\title{
Tuberculosis: When Difficult to Select Treatment Regimen
}

\author{
Ariful Basher \\ Registrar, Infectious \& Tropical Medicine Department, Mymensingh Medical College Hospital, Mymensingh, Bangladesh; Email: \\ ariful.dr@gmail.com
}

Pulmonary tuberculosis decreased remarkably by anti-tuberculous chemotherapy, but we have had a lot of patients associated with sequelae who need medical treatment for many symptoms. We also have a lot of patients diagnosed as an extra pulmonary tuberculosis where diagnosed is usually based on histological findings and treatment duration was based on expert opinion. Besides, some patients presented with extensive tuberculosis or with so many complications that predict nonresponsive to CAT-1 tuberculosis drugs. Moreover, in these days the delayed detection of pulmonary tuberculosis that is still an important problem may cause new sequelae.

Tuberculosis some time may be beyond the category based management due to lack of confirmatory diagnostic tools and facilities, especially in resource constrain country like Bangladesh. Many CAT-1 non responsive patients presented with so many chest complication like fibrosis, bronchectasis, collapse, cavity etc that was difficult to consider whether patients still have tuberculosis especially when existing facilities failed to identify.
Lymph node or wound infection tuberculosis generally difficult to identify the bacilli are many times non responsive to usual tuberculosis treatment regimen. Existing guideline are sometimes contradict the sequence of management protocol with the real need of the patients. Unjustified or misuse of tuberculosis drugs are not uncommon especially when clinician failed to correlate the patients situation and guideline suggestions. Many bone or spine tuberculosis patients had history taking anti tuberculous drugs more than two years with little improvement. Surgical debridement or Prophylaxis might be thinking in very special cases where no decision can be made.

I pointed out the problems of complicated presentation of tuberculosis where extensive investigations and judicious chosen of regimen are needed. These problems may be beyond the category of tuberculosis study, but are extremely important for infectious diseases physicians.

[Bangladesh Journal of Infectious Diseases 2015;2(2):27]

[Citation: Basher A. Tuberculosis; When Difficult to Select Treatment Regimen. Bangladesh J Infect Dis 2015;2(2):27] 